Preprint DSF-8/94

April 1994

\title{
Semiclassical Gravitational Effects in de Sitter Space at Finite Temperature
}

\author{
D.V. Fursaev ${ }^{1,2}$ and G. Miele ${ }^{2}$ \\ ${ }^{1}$ Laboratory of Theoretical Physics, Joint Institute for Nuclear Research, \\ Head Post Office, P.O.Box 79, Moscow, Russia \\ ${ }^{2}$ Dipartimento di Scienze Fisiche, Università di Napoli - Federico II -, and INFN \\ Sezione di Napoli, Mostra D'Oltremare Pad. 19, 80125, Napoli, Italy
}

\begin{abstract}
In the framework of finite temperature conformal scalar field theory on de Sitter space-time the linearized Einstein equations for the renormalized stress tensor are exactly solved. In this theory quantum field fluctuations are concentrated near two spheres of the de Sitter radius, propagating as light wave fronts. Related cosmological aspects are shortly discussed. The analysis, performed for flat expanding universe, shows exponential damping of the back-reaction effects far from these spherical objects. The obtained solutions for the semiclassical Einstein equations in de Sitter background can be straightforwardly extended also to the anti-de Sitter geometry.
\end{abstract}




\section{Introduction}

In the modern picture of the early universe the exponential expansion period is known to be successful in solving a number of important problems occurring in the standard cosmology. During this inflationary phase, described by de Sitter geometry, quantum field effects played an essential role for subsequent formation of the present universe. In this framework, the knowledge of the corresponding quantum state, originating from Planck epoch, is important to reconstruct the phase transitions pattern of the inflation. One of the possibilities generally used [1], [2] is to consider this quantum state as a de Sitter invariant vacuum experienced by a freely moving observer as a thermal bath at the Hawking temperature $\beta_{H}^{-1}$ [3].

A wider class of quantum states, associated in static de Sitter coordinates to a thermal equilibrium at arbitrary temperature $\beta^{-1}$, has been investigated in our previous work [4. In the present letter we extend this analysis studying the back-reaction on de Sitter geometry in these quantum states.

For simplicity we restrict our consideration to massless conformally invariant scalar theory where the finite temperature Green function, globally defined on the whole spacetime, can be exactly found. However, the corresponding renormalized stress tensor for arbitrary temperatures includes a traceless part singular on the horizon surface of two antipodal static coordinate systems, where thermal equilibrium is introduced. This extremely large energy density implies that, very close to the horizon, physical quantities can be computed only in the framework of quantum gravity, theory which would provide a natural short distance cut-off 円. Thus, the singular part of the stress tensor can be interpreted as matter distribution sharply concentrated over two spherical shells of radius $r_{0}$ (horizon size) that propagate through the de Sitter hyperboloid as light wave fronts. So far as, apart from the rest space-time, the quantum field inside the domains bounded by the shells is in thermal equilibrium, we will refer to these spherical 3-d regions as bubbles 2], that can be characterized by temperature $\beta^{-1}$, internal energy, and entropy.

\footnotetext{
1 Note that recently much attention has been paid to similar divergences appearing in statistical mechanical computations of black hole entropy [5]- [8], when temperature different from the Hawking one has to be introduced to obtain the derivative of the partition function. Possible role of superstring theory, providing such fundamental cut-off, is discussed in [7].

${ }^{2}$ It is worth reminding that these bubbles are different from the topological defects nucleated during
} 
This letter is organized as follows: in section 2 we compute the renormalized stress tensor at finite temperature $\beta \neq \beta_{H}$, and discuss its properties inside and outside the bubbles. In section 3 this tensor is used to evaluate, in the linear approximation, the backreaction effects. The model of the flat expanding de Sitter universe is then considered in section 4 to study the cosmological implications of the chosen quantum state. Finally, we give our conclusions and remarks.

\section{The quantum state and stress tensor}

Let us determine the average value of the energy momentum tensor for conformally invariant scalar field theory $<\hat{T}_{\mu}^{\nu}>_{\beta} \equiv T_{\mu}^{\nu}(\beta)$ in static de Sitter space, where $\beta$ is the inverse temperature of the system. This tensor can be decomposed as

$$
T_{\mu}^{\nu}(\beta)=\tilde{T}_{\mu}^{\nu}(\beta)+\frac{1}{4} \delta_{\mu}^{\nu} T
$$

where $\tilde{T}_{\mu}^{\nu}(\beta)$ is the traceless part, and $T \equiv T_{\mu}^{\mu}(\beta)$ is the local conformal anomaly that does not depend on the quantum state of the system [9]. In particular, for de Sitter space

$$
T=\frac{1}{240 \pi^{2} r_{0}^{4}}
$$

( $r_{0}$ stands for the de Sitter radius), and due to the conservation law $\tilde{T}_{\mu ; \nu}^{\nu}=0$, the traceless part has only one independent component. Consequently, to find all its components it is sufficient to calculate only one of them.

It is worth reminding that static de Sitter metric $g_{\mu \nu}$ reads

$$
d s^{2}=g_{\mu \nu} d x^{\mu} d x^{\nu}=\left(1-r^{2} / r_{0}^{2}\right) d t^{2}-\left(1-r^{2} / r_{0}^{2}\right)^{-1} d r^{2}-r^{2}\left(d \theta^{2}+\sin ^{2} \theta d \varphi^{2}\right),
$$

where $x^{\mu} \equiv(t, r, \theta, \varphi)$. In this space the temporal component of the stress tensor $\tilde{T}_{t}^{t}(\beta)$ can be obtained observing that [10]

$$
<\hat{T}_{t}^{t}>_{\beta}-<\hat{T}_{t}^{t}>_{\beta=\infty}=\frac{\pi^{2}}{30} \frac{\beta^{-4}}{g_{t t}^{2}} .
$$

The last equation can be also rewritten in the following form

$$
<\hat{T}_{t}^{t}>_{\beta}=<\hat{T}_{t}^{t}>_{\beta_{H}}+\frac{\pi^{2}}{30}\left(1-\frac{\beta^{4}}{\beta_{H}^{4}}\right) \frac{\beta^{-4}}{g_{t t}^{2}},
$$

phase transitions 
where $\beta_{H} \equiv 2 \pi r_{0}$ denotes the inverse Hawking temperature. Hence, due to the fact that for $\beta=\beta_{H}$ the stress tensor is completely anomalous $\left(\tilde{T}_{\mu}^{\nu}\left(\beta_{H}\right)=0\right)$, we have

$$
\tilde{T}_{t}^{t}(\beta)=<\hat{T}_{t}^{t}>_{\beta}-<\hat{T}_{t}^{t}>_{\beta=\beta_{H}}=\frac{\pi^{2}}{30}\left(1-\frac{\beta^{4}}{\beta_{H}^{4}}\right) \frac{\beta^{-4}}{g_{t t}^{2}}
$$

This enables to obtain the stress tensor in the form

$$
<\hat{T}_{\mu}^{\nu}>_{\beta}=\frac{\pi^{2}}{30}\left(1-\frac{\beta^{4}}{\beta_{H}^{4}}\right) \frac{\beta^{-4}}{g_{t t}^{2}} \operatorname{diag}\left(1,-\frac{1}{3},-\frac{1}{3},-\frac{1}{3}\right)+\frac{1}{960 \pi^{2} r_{0}^{4}} \delta_{\mu}^{\nu}
$$

that coincides at zero temperature with the known result for the static conformal vacuum [11.

As one can see from (2.7) the traceless part dominates near the horizon and corresponds for $\beta<\beta_{H}$ to the energy momentum tensor of a gas of massless scalar particles [12]. In this case, the energy density has the usual Planck form and results to be

$$
\tilde{T}_{t}^{t}(\beta)=\frac{\pi^{2}}{30} \tilde{\beta}^{-4}
$$

where $\tilde{\beta}^{-1}=\beta^{-1}\left(1-\beta^{4} / \beta_{H}^{4}\right)^{1 / 4} g_{t t}^{-1 / 2}$ plays the role of a local, redshifted temperature. The similar structure for the energy density in finite temperature Rindler space is given in 13.

Let us point out that (2.7) can be obtained by the standard procedure from the finite temperature Green function given by [10]

$$
G_{\beta}\left(x, x^{\prime}\right)=i \frac{\left[\left(1-r^{2} / r_{0}^{2}\right)\left(1-r^{\prime 2} / r_{0}^{2}\right)\right]^{-1 / 2}}{2 \beta_{H} \beta \sinh \alpha_{1}} \frac{\sinh \left(\alpha_{1} \beta_{H} / \beta\right)}{\cosh \left(\alpha_{1} \beta_{H} / \beta\right)-\cosh \left[\left(t-t^{\prime}\right) \beta_{H} / r_{0} \beta\right]},
$$

$\left(\cosh \alpha_{1}=\left[\left(r_{0}^{2}-r^{2}\right)\left(r_{0}^{2}-r^{\prime 2}\right)\right]^{-1 / 2}\left\{r_{0}^{2}-r r^{\prime}\left[\cos \theta \cos \theta^{\prime}+\sin \theta \sin \theta^{\prime} \cos \left(\varphi-\varphi^{\prime}\right)\right]\right\}\right)$, with the subtraction of its value corresponding to the Hawking temperature. Although expression (2.9) is defined only in the region bounded by the horizon surface, it can be extended to the total de Sitter space-time. In terms of conformal diagram, figure 1, where static coordinate system (2.3) maps into the region (a), it is easy to prove that $G_{\beta}\left(x, x^{\prime}\right)$ can be smoothly continued from the region (a) into the casually connected domains (c) or (d), by moving one by one its arguments through the horizon surface. Then extending this procedure from (c) or (d) to (b) we obtain a globally defined two-point Green function, smooth everywhere for not coinciding arguments. Such procedure completely fixes in all 
the de Sitter space-time the quantum state, whose properties can be derived from the structure of the associated global energy momentum tensor. This global stress tensor as opposite to the Green function is singular on the horizon surface, which means that close to the horizons our semiclassical approach is not applicable and the complete theory of quantum gravity should be used for computing the quantum effects.

As we have already mentioned one can interpret the energy singularity as a matter distribution over the surface of two spherical bubbles having the horizon size $r_{0}$, and propagating as fronts of light waves. Regions (a) and (b) represent their internal regions, where the stress tensor has identical forms, (2.7). In the external regions (c), (d) the metric (2.3) changes to

$$
d s^{2}=\left(r^{2} / r_{0}^{2}-1\right)^{-1} d r^{2}-\left(r^{2} / r_{0}^{2}-1\right) d t^{2}-r^{2}\left(d \theta^{2}+\sin ^{2} \theta d \varphi^{2}\right)
$$

and describes an expanding (or shrinking) universe where $r$ now plays the role of time and $t$ has the meaning of a spatial coordinate. In these case, the horizon surfaces are removed to spatial infinities $(t= \pm \infty)$, and the structure of the stress tensor can be found on the base of (2.7). It follows immediately that in these domains $\tilde{T}_{\mu}^{\nu}(\beta)$ corresponds to a non equilibrium thermal system. Although the energy flows from the infinities $(t= \pm \infty)$ are absent, the energy density decreases to the vacuum one due to the factor $\left[g_{t t}(r)\right]^{-2}$ in according with adiabatic expansion of this universe.

A remark concerning the thermodynamical parameters of the bubbles like their energy, and entropy it is worth being done here. These quantities can be computed in two ways: through the local energy $T_{t}^{t}(\beta)$ [6], or in terms of the one-loop partition function 迎. The first method cannot be straightforwardly applied due to the divergence of the local energy and entropy at the horizon, whereas the other one gives a finite answer under $\zeta$-function regularization [4]. This fact might be understood in terms of renormalization of bubbles surface energy, which can cancel the local divergence. To partially support the above statement we refer to the analysis carried out for manifolds with conical singularities [14]. Such kind of defects is inherent to "gravitational instantons" associated to static de Sitter space $(2.3)$ at $\beta \neq \beta_{H}$ [四]. 


\section{The semiclassical analysis}

Consider now changing of de Sitter geometry induced by the vacuum polarization in the given quantum states. Einstein equations in semiclassical approximation are known to read as 9

$$
R_{\mu \nu}(\bar{g})-\frac{1}{2} R(\bar{g}) \bar{g}_{\mu \nu}+\Lambda \bar{g}_{\mu \nu}+\alpha{ }^{(1)} H_{\mu \nu}(\bar{g})+\beta^{(2)} H_{\mu \nu}(\bar{g})=-8 \pi G<\hat{T}_{\mu \nu}>_{\beta} \quad .
$$

Here $\bar{g}_{\mu \nu}=g_{\mu \nu}+h_{\mu \nu}$ indicates the solution of (3.11) including quantum corrections $h_{\mu \nu}$ to de Sitter metric $g_{\mu \nu}$, and $\Lambda=3 / r_{0}^{2}$ is the cosmological constant. As for the additional tensors ${ }^{(1)} H_{\mu \nu}$ and ${ }^{(2)} H_{\mu \nu}$ that appear due to the quantum corrections, and are defined in terms of the geometrical quantities [9], we will neglect them in the further analysis assuming the unknown constants $\alpha, \beta$ in (3.11) to be equal to zero. Then, expanding (3.11) up to the first order in the metric perturbation $h_{\mu \nu}$, we have

$h_{\mu ; \nu \alpha}^{\alpha}+h_{\nu ; \mu \alpha}^{\alpha}-h_{\alpha ; \mu \nu}^{\alpha}-h_{\mu \nu ; \alpha}^{; \alpha}+\frac{6}{r_{0}^{2}} h_{\mu \nu}+\left(h_{\beta ; \alpha}^{\beta ; \alpha}-h_{\alpha ; \beta}^{\beta ; \alpha}-\frac{3}{r_{0}^{2}} h_{\alpha}^{\alpha}\right) g_{\mu \nu}=16 \pi G<\hat{T}_{\mu \nu}>_{\beta}$

An essential progress in solving these equations can be achieved observing that the anomalous part of the renormalized stress tensor in the r.h.s. of (3.12) only gives rise to a redefinition of the de Sitter radius $r_{0}$. Taking into account this trivial effect we can use

instead of the total tensor only its traceless part $\tilde{T}_{\mu}^{\nu}(\beta)$. Thus, after a little transformation, the initial equations take the form

$$
h_{\mu \nu ; \alpha}^{; \alpha}+\frac{2}{r_{0}^{2}} h_{\mu \nu}=-16 \pi G \tilde{T}_{\mu \nu}(\beta),
$$

where we imposed the gauge conditions $h_{\mu \nu}^{; \nu}=0, h_{\mu}^{\mu}=0$ (the latter is compatible with the dynamical equations and fixes the residual gauge freedom). Besides this, the property of de Sitter space for which $R_{\lambda \nu \mu \alpha}=r_{0}^{-2}\left(g_{\lambda \mu} g_{\nu \alpha}-g_{\lambda \alpha} g_{\nu \mu}\right)$ has been used in (3.13).

To proceed in our analysis, it is worth reminding that in the given quantum state $\left(\beta \neq \beta_{H}\right)$ the total de Sitter symmetry $S O(1,4)$ is broken to the subgroup $T_{1} \times S O(3)$, where $T_{1}$ stands for de Sitter boosts associated with translations along the coordinate $t$. This enables us to restrict the degrees of freedom of $h_{\mu \nu}$, to the only nonzero components 
$h_{t t}(r), h_{r r}(r), h_{t r}(r)=h_{r t}(r)$, and $h_{i k} \equiv-a(r) r^{2} \gamma_{i k}$, where the indexes $i, k$ are referred to the $\theta, \varphi$ coordinates, and $\gamma_{i k}$ is the metric on $S^{2}$ (in accordance with (2.3) $g_{i k}=-r^{2} \gamma_{i k}$ ).

Due to the residual symmetry and gauge conditions imposed, there is only one independent equation among (3.13). After some algebra one can show that in static coordinates it reduces to a second order differential equation for $h_{r r}$

$$
\left[4(1-y) \frac{d^{2}}{d y^{2}}-\frac{26 y-16}{y} \frac{d}{d y}-\frac{28 y-8}{y^{2}}\right] h_{r r}(y)=-\frac{K(\beta)}{y^{4}},
$$

written in terms of the variable $y(r) \equiv 1-r^{2} / r_{0}^{2}$ and a constant

$$
K(\beta) \equiv \frac{G}{90 \pi r_{0}^{2}}\left(1-\frac{\beta_{H}^{4}}{\beta^{4}}\right) .
$$

Remarkably, the homogeneous equation associated to (3.14) admits two simple solutions: $y^{-2}$, and $\left[y^{2}(1-y)^{3 / 2}\right]^{-1}$. Thus the general integral of (3.14) can be given in the following form

$$
\begin{aligned}
h_{r r}(r) & =\frac{K(\beta)}{4 y^{2}(r)}\left\{\frac{2}{3}+\frac{2}{1-y(r)}+\frac{1}{[1-y(r)]^{3 / 2}} \log \left[\frac{1-\sqrt{1-y(r)}}{1+\sqrt{1-y(r)}}\right]\right\} \\
& +\frac{A}{y^{2}(r)}+\frac{B}{y^{2}(r)[1-y(r)]^{3 / 2}} .
\end{aligned}
$$

It is worth mentioning that coordinate systems in de Sitter spaces, (2.3), (2.10), can be treated on equal footing if one adds a small imaginary part to de Sitter radius $r_{0} \rightarrow r_{0}+i \epsilon$. This regularization preserves the structure of all the equations in both quantum and classical theory. It removes the singularity at the horizons in such a way that (2.10), and (2.3) can be unified in a single expression for the metric valid for $0 \leq r<\infty$. However, although such regularization allows to define the solution in all the region $0 \leq r<\infty$, the integration constants $A$ and $B$ cannot be chosen to be equal in all this interval. Indeed, if $A$ and $B$ are fixed in some way in the inner domain (a), then after passing in the external region, say (c), one obtains due to the logarithm complex values for the function $h_{r r}(r)$. It means that integration constants for external and internal problems in the given semiclassical approach should be found independently, on the base of additional physical motivations.

In the static regions $A$ and $B$ can be chosen from the condition $h_{\mu \nu}(r)=0$ at $r=0$ that corresponds to the natural assumption for the vacuum effects to disappear in the flat limit 
$r / r_{0}<<1$, when the background space converts into Minkowski one. As for the external domains, one can require for the perturbed metric to approach the de Sitter metric in the limit $r \rightarrow \infty$, where local quantum effects disappear (see (2.7)). The components $h_{t t}$, $h_{i k}$, can be obtained from (3.16) using constraints. On the other hand, the non diagonal element $h_{t r}$, fixed by the condition $h_{t \nu}{ }^{\nu}=0$, turns out to be completely independent of the stress tensor and other components. For this reason, we put it to be zero everywhere, which inside the horizon can be also justified by claiming its regularity at $r=0$.

By virtue of the expression (3.16) and conditions chosen we can represent all the non zero components of the metric perturbations in the following form

$$
\begin{aligned}
h_{t t}(r) & =\frac{K(\beta)}{4}\left[2-\frac{4 r^{2}}{3 r_{0}^{2}}+\frac{r_{0}}{2 r} \log \left(\frac{r_{0}-r}{r_{0}+r}\right)^{2}\right]+A\left(3-\frac{2 r^{2}}{r_{0}^{2}}\right)+B \frac{r}{r_{0}}, \\
h_{r r}(r) & =\frac{K(\beta)}{4 y^{2}(r)}\left[\frac{2}{3}+\frac{2 r_{0}^{2}}{r^{2}}+\frac{r_{0}^{3}}{2 r^{3}} \log \left(\frac{r_{0}-r}{r_{0}+r}\right)^{2}\right]+\frac{A}{y^{2}(r)}+B \frac{r_{0}^{3}}{y^{2}(r) r^{3}}, \\
a(r) & =\frac{K(\beta)}{4}\left[-\frac{2}{3}+\frac{r_{0}^{2}}{r^{2} y(r)}+\frac{r_{0}^{3}}{4 r^{3}} \log \left(\frac{r_{0}-r}{r_{0}+r}\right)^{2}\right]-A+B \frac{r_{0}^{3}}{2 r^{3}} .
\end{aligned}
$$

Following the previously mentioned requirements, the integration constants $A$ and $B$ have to be put equal to zero for $0 \leq r<r_{0}$, whereas for $r_{0}<r<\infty$ we have $A=-K(\beta) / 6$. As far as the value of $B$ in the external region is concerned, it is left undetermined. This fact can be understood reminding that in the approximated approach of semiclassical theory two regions are separated by an infinite barrier, whereas in the correct theory both solutions should be matched at the horizon, so fixing the remaining constant. Note also that solutions (3.17)- 3.19) are valid only in the region where the perturbation expansion is reliable. In terms of the Planck length $l_{P l} \equiv \sqrt{G}$, this region results to be $\left|r-r_{0}\right|>>\left|1-\beta_{H}^{4} / \beta^{4}\right|^{1 / 2} l_{P l}$. Therefore, if the de Sitter radius $r_{0} \approx l_{P l}$ the present analysis is not applicable to the bubble interior.

Finally, we observe that solutions (3.17)-(3.19) of semiclassical Einstein equations in de Sitter background, can be immediately extended to anti-de Sitter space just substituting $r_{0}$ with $i r_{0}$. This is connected to the fact that the renormalized stress tensor at finite temperature in anti-de Sitter space has the same structure of de Sitter one, once a complex scalar field with Neumann and Dirichlet boundary conditions for its two independent 
components is chosen 15.

\section{Gravitational effects}

We discuss now the properties of the space taking into account quantum corrections 3.17) - (3.19) to de Sitter metric. It can be done considering the different models of de Sitter space corresponding to flat, open or closed expanding universes. For sake of simplicity we restrict in this letter our analysis to the flat model, which is generally used for cosmological applications. A more complete study will be given in a forthcoming paper [16].

The corresponding metric has the familiar form

$$
d s^{2}=d \tau^{2}-e^{2 \tau / r_{0}}\left(d \xi^{2}+\xi^{2} d \Omega^{2}\right) \quad, \quad 0 \leq \xi<\infty \quad,
$$

$\left(d \Omega^{2}=d \theta^{2}+\sin ^{2} \theta d \varphi^{2}\right.$ is the line element on a sphere). This system of coordinates maps only a half of the de Sitter hyperboloid. For a more clear representation we assume it to cover regions (a) and (c) on figure 1. In this case, one of the singularities of the global stress tensor is placed inside the universe (4.20), in such a way that bubble's center corresponds to the origin of coordinates. At the same time, the other one is at spatial infinity $(\xi \rightarrow \infty)$. The map between (4.20) and (2.3) or (2.10) can be written as follows

$$
t=\tau-\frac{r_{0}}{4} \log \left(1-\frac{\xi^{2}}{r_{0}^{2}} e^{2 \tau / r_{0}}\right)^{2} \quad, \quad r=\xi e^{\tau / r_{0}} .
$$

Note that by definition, the shell of the bubble coincides with de Sitter horizon thus its size remains unchanged during inflation ${ }^{3}$, but due to the expansion every point, once inside it, will move away. Near the matter distribution surface $\xi_{0}=r_{0} \exp \left(-\tau / r_{0}\right)$ the metric 4.20 ) changes to

$$
d s^{2}=\left(1+h_{\eta \eta}\right) d \eta^{2}+2 h_{\eta \xi} d \eta d \xi-\left(e^{2 \eta / r_{0}}-h_{\xi \xi}\right) d \xi^{2}-e^{2 \eta / r_{0}} \xi^{2}(1+a) d \Omega^{2}
$$

where we introduced $\eta$ instead of $\tau$ to indicate that now it is not the proper time. The metric perturbations $h_{\eta \eta}, h_{\eta \xi}$ and $h_{\xi \xi}$ follow from (3.17), (3.18) and map (4.21), where $\tau$ is replaced by $\eta$

$$
h_{\eta \eta}(\eta, \xi)=\frac{1}{y^{2}} h_{t t}+\frac{\xi^{2}}{r_{0}^{2}} e^{2 \eta / r_{0}} h_{r r}
$$

\footnotetext{
${ }^{3} \mathrm{~A}$ discussion about this point is given in section 5 .
} 


$$
\begin{aligned}
& h_{\eta \xi}(\eta, \xi)=\frac{\xi}{r_{0}} e^{2 \eta / r_{0}}\left(\frac{1}{y^{2}} h_{t t}+h_{r r}\right) \\
& h_{\xi \xi}(\eta, \xi)=e^{2 \eta / r_{0}}\left(\frac{\xi^{2}}{r_{0}^{2} y^{2}} e^{2 \eta / r_{0}} h_{t t}+h_{r r}\right) .
\end{aligned}
$$

In Eqs. (4.22)-(4.25) all the functions $y, a, h_{t t}$ and $h_{r r}$ are supposed to be expressed in terms of $\eta$ and $\xi$. So far as $\eta$ does not represent the proper time we need an additional transformation to pass to comoving coordinates $(\tau, x, \theta, \varphi)$, similar to (4.20),

$$
d s^{2}=d \tau^{2}-e^{2 \tau / r_{0}}\left[\left(1+h_{x x}(x, \tau)\right) d x^{2}+x^{2}\left(1+h_{\Omega \Omega}(x, \tau)\right) d \Omega^{2}\right] \equiv d \tau^{2}-\gamma_{i j} d x^{i} d x^{j}
$$

with $i, j=1,2,3$, corresponding to $x, \theta$ and $\varphi$ respectively. The above transformation reads

$$
\eta=\tau+\eta_{1}(\tau, x) \quad, \quad \xi=x+\xi_{1}(\tau, x)
$$

and the functions $\eta_{1}$ and $\xi_{1}$ obey to the following differential equations

$$
2 \frac{\partial \eta_{1}}{\partial \tau}+h_{\eta \eta}=0 \quad, \quad e^{2 \tau / r_{0}} \frac{\partial \xi_{1}}{\partial \tau}-h_{\eta \xi}-\frac{\partial \eta_{1}}{\partial x}=0
$$

In terms of these functions the metric corrections $h_{x x}$ and $h_{\Omega \Omega}$ result to be

$$
h_{x x}=2 \frac{\eta_{1}}{r_{0}}+2 \frac{\partial \xi_{1}}{\partial x}-e^{-2 \tau / r_{0}} h_{\xi \xi} \quad, \quad h_{\Omega \Omega}=2 \frac{\eta_{1}}{r_{0}}+2 \frac{\xi_{1}}{x}+a
$$

It is worth observing that the integration constant for $\xi_{1}$, obtained from (4.28), is not relevant because it can always be changed by redefinition of $x$ coordinate in (4.26). As far as $\eta_{1}$ is concerned, its value is fixed, up to an inessential numerical constant, by requiring that metric components (4.26) depend only on the distance $r=x \exp \left(\tau / r_{0}\right)$ from the center of the perturbation.

The metric components can be readily obtained from (4.28) on the total space $(0 \leq$ $r<r_{0}$ and $\left.r_{0}<r<\infty\right)$. For sake of brevity we only present the expressions for the outside region to investigate the metric asymptotic at infinity. They read

$$
h_{\Omega \Omega}=K(\beta)\left[-\frac{5}{4} \log \left(\frac{r^{2}}{r^{2}-r_{0}^{2}}\right)+\frac{r_{0}^{3}}{8 r^{3}} \log \left(\frac{r-r_{0}}{r+r_{0}}\right)+\frac{r_{0}^{2}}{4 r^{2}} \frac{2 r_{0}^{2}-3 r^{2}}{r_{0}^{2}-r^{2}}\right] \text {, }
$$




$$
h_{x x}=K(\beta)\left[-\frac{5}{4} \log \left(\frac{r^{2}}{r^{2}-r_{0}^{2}}\right)+\frac{r_{0}^{2}}{4 r^{2}} \frac{r_{0}^{2}-3 r^{2}}{r_{0}^{2}-r^{2}}\right],
$$

where the undefined constant $B$ was neglected, because it does not affect the long-range behavior $\left(r \gg r_{0}\right)$ of perturbations. By using (4.30) and (4.31) one can see that gravitational field far from the bubbles decreases like $r^{-2}$. It corresponds, due to the expansion, to an exponential falling with time $\tau$ for a point of given $x$.

The effects of the quantum corrections to the metric (4.20) can be expressed in another way by computing the spatial curvature $R^{(3)}(\gamma)$ of the universe (4.26)

$$
\begin{gathered}
R^{(3)}(\gamma)=h_{i ; j}^{i ; j}-h_{i ; j}^{j ; i} i=\frac{2}{x^{2}} e^{-2 \tau / r_{0}} \frac{d}{d x}(x f(x, \tau)), \\
f(x, \tau) \equiv \frac{d}{d x}\left(x h_{\Omega \Omega}\right)-h_{x x}=3 a+x \frac{d}{d x} a .
\end{gathered}
$$

Remarkably, due to (4.33) it turns out to be completely independent of the constant $B$ and takes the simple analytical forms

$$
\left.R^{(3)}(\gamma)\right|_{r<r_{0}}=K(\beta) \frac{3 r_{0}^{2}-r^{2}}{\left(r^{2}-r_{0}^{2}\right)^{2}} \quad,\left.\quad R^{(3)}(\gamma)\right|_{r>r_{0}}=K(\beta) \frac{r_{0}^{2}}{r^{2}} \frac{r^{2}+r_{0}^{2}}{\left(r^{2}-r_{0}^{2}\right)^{2}}
$$

for inside and outside regions respectively. By definition (3.15) of $K(\beta)$ it follows that curvature is negative if the bubble temperature $\beta^{-1}$ higher then the Hawking one $\beta_{H}^{-1}$, and changes the sign when $\beta^{-1}<\beta_{H}^{-1}$. Moreover, its value at $r=0$ does not depend on time $\left.R^{(3)}(\gamma)\right|_{r=0}=3 K(\beta) r_{0}^{-2}$, whereas in external region $R^{(3)}$ decreases as $r^{-4}$. This long range behaviour of the gravitational field is connected to the massless nature of the chosen scalar field. For massive matter fields one can expect that the gravitational effects will be exponentially smeared, on a length of the order of the inverse mass, outside the bubble shell.

\section{Conclusions and remarks}

In this letter the local properties of thermal states for a quantum field in de Sitter spacetime have been investigated. We interpret the known singularity of renormalized stresstensor as a matter distribution located on two spherical surfaces (boundaries of bubbles) moving far away one from each other. Such domains, characterized by temperature, entropy, and etc., give rise to a number of semiclassical effects. 
To find the gravitational field produced by these perturbations, the linearized Einstein equations on de Sitter background have been exactly solved for stress tensor of a conformal scalar field at finite temperature. The computations show the gravitational effects are weak far from the bubbles, and in the expanding universe they are exponentially damped.

In realistic cosmological models both the radius and thermodynamical properties of the bubbles might change during the universe evolution. Note that external region of static coordinates (2.3) can be considered as a "black hole" absorbing "information" from the inner domain due to decays there of unstable field configurations of the Higgs scalars. As a result of this process, the bubble size, associated to the horizon, grows up and achieves cosmological values determined by the inverse of the Hubble constant,

$H^{-1} \approx 10^{28} \mathrm{~cm}$ at the present. However, due to the exponential damping, the presence of these inhomogeneous regions does not seem to affect the observable part of the universe, provided if it is far enough from them. We confine the present letter to these short remarks, leaving more complete analysis of the cosmological aspects of such theory to a forthcoming paper [16].

Finally, it is worth observing that the solutions obtained for the semiclassical Einstein equations in de Sitter background can be straightforwardly extended to the anti-de Sitter geometry. In this case in fact, the stress tensor has the same structure of the de Sitter one, once a complex scalar field with appropriate boundary conditions is chosen [15].

We would like to thank Bruce Allen and Sergey Solodukhin for their interesting remarks. This work was supported in part by International Science Foundation (Soros) Grant No. Ph1-0802-0920.

\section{References}

[1] B. Allen, Nucl. Phys. B226 (1983) 228; B. Allen, Ann. Phys. 161 (1985) 152.

[2] F. Buccella, G. Esposito, G. Miele, Class. Quantum Grav. 9 (1992) 1499; G. Esposito, G. Miele and L. Rosa, submitted to Class. Quantum Grav.

[3] G.W. Gibbons and S.W. Hawking, Phys. Rev. D15 (1977) 2738.

[4] D.V. Fursaev and G. Miele, Phys. Rev. D49 (1994) 987. 
[5] C. Callan and F. Wilczek, On geometric entropy, preprint IASSNS-HEP-93/87.

[6] J.S. Dowker, Remarks on geometric entropy, preprint MUTP/94/2.

[7] L. Susskind and J. Uglum, Black hole entropy in canonical quantum gravity and superstring theory, preprint SU-ITP-94-1.

[8] T. Jacobson, Black hole entropy and induced gravity, preprint gr-qc/9404039.

[9] N.D. Birrell and P.C.W. Davis, Quantum fields in curved space (Cambridge University Press, 1982).

[10] J.S. Dowker, Phys. Rev. D18 (1978) 1856.

[11] P. Candelas and J.S. Dowker, Phys. Rev. D19 (1979) 2902.

[12] L.D. Landau and E.M. Lifshitz, Statistical Physics (Pergamon Press, London - Paris, 1958).

[13] J.S. Dowker, "Quantum field theory around conical deffects" in The formation and evolution of cosmic strings edited by G.Gibbons et al., Cambridge University Press, 1990.

[14] D.V. Fursaev, Class. Quantum Grav. 11 (1994) 1.

[15] B. Allen, A. Folacci and G.W. Gibbons, Phys. Lett. B 189 (1987) 304.

[16] D.V. Fursaev and G. Miele, in progress. 


\section{Figure caption}

Figure 1: In this diagram left and right edges have to be identified. Regions (a) and (b) correspond to static coordinates with $0 \leq r<r_{0}$, separated by light cones from (c) and (d) regions where $r_{0}<r<+\infty$. Dashed lines in regions (a) and (b) represent the trajectories of a particle and its antipod being in the coordinate origins. 
This figure "fig1-1.png" is available in "png" format from: http://arxiv.org/ps/gr-qc/9404048v1 